\title{
Численное интегрирование системы связанных нелинейных уравнений Шредингера
}

\author{
$\underline{\text { А.Ж. Сахабутдинов }}{ }^{1, *}$, В.И. Анфиногентов ${ }^{1}$, О.Г. Морозов ${ }^{1}$, \\ В.А. Бурдин ${ }^{2}$, А.В. Бурдин ${ }^{2}$ \\ ${ }^{1}$ Казанский национальны исследовательский технический университет им. А.Н. Туполева-КАИ \\ ${ }^{2}$ Поволжский государственный университет телекоммуникаций и информатики
}

"E-mail: kazanboy@yandex.ru

DOI: 10.31868/RFL2020.83-84

Совершенствование фемтосекундных лазеров и их все более широкое применение делают актуальными решение задач доставки ультракороткого оптического импульса с заданными параметрами [1] до заданной точки и, как следствие, исследования распространения ультракоротких импульсов большой мощности по оптическим волокнам, в том числе и по оптическим волокнам с сохранением поляризации. В [2] достаточно подробно описан эксперимент по передаче оптического импульса длительностью 12 фс по одномодовому оптическому волокну с сохранением поляризации на расстояние 2,5 мм. В этих же работах представлены результаты расчетов импульсного отклика на выходе оптического волокна, полученные, кроме прочих, и методом конечных разностей во временной области без учета и с учетом хроматической дисперсии третьего порядка и рассеяния Рамана. Описывая распространение оптических импульсов в одномодовом оптическом волокне с сохранением поляризации, необходимо учитывать распространение двух ортогонально-поляризованных мод.

В работе система связанных уравнений Шредингера приведена к виду, описывающему передачу ультракороткого оптического импульса при распространении в одномодовом оптическом волокне с сохранением поляризации двух ортогонально-поляризованных мод. Система уравнений записывается в конечноразностных соотношениях, при этом проводится разделение формы записи линейных и нелинейных слагаемых. Запись линейных слагаемых осуществляется по неявной схеме, а нелинейных по явной конечно-разностной схеме. Подход позволил на каждом шаге интегрирования разделить систему уравнений Шрёдингера на две системы линейных уравнений относительно каждой моды.

В общем виде эволюция коротких оптических импульсов в оптическом волокне при распространении в нем нескольких мод описывается системой связанных нелинейных уравнений Шредингера:

$$
\left\{\begin{aligned}
\frac{\partial A_{i}}{\partial z} & =-\frac{\alpha_{i}}{2} A_{i}-\beta_{1, i} \frac{\partial A_{i}}{\partial t}-j \frac{\beta_{2, i}}{2} \frac{\partial^{2} A_{i}}{\partial t^{2}}+\frac{\beta_{3, i}}{6} \frac{\partial^{3} A_{i}}{\partial t^{3}}+ \\
& +j \gamma_{i} A_{i} \sum_{m=1}^{M} C_{i, m}\left|A_{m}\right|^{2}-\frac{\gamma_{i}}{\omega_{0, i}} \sum_{m=1}^{M} B_{i, m} \frac{\partial\left(\left|A_{m}\right|^{2} A_{i}\right)}{\partial t}-j \gamma_{i} T_{R} A_{i} \sum_{m=1}^{M} B_{i, m} \frac{\partial\left(\left|A_{m}\right|^{2}\right)}{\partial t} \\
i & =\overline{1, M}
\end{aligned}\right.
$$

где $A_{i}$ - комплексные огибающие оптического импульса $i$-ой моды; $\alpha_{i}$ - коэффициент затухания $i$-ой моды; $\beta_{1, i}, \beta_{2, i}, \beta_{3, i}$ - параметры дисперсии первого, второго и третьего порядков $i$-ой моды соответственно; $\gamma_{i}$ - параметр нелинейности для $i$-ой моды; $C_{i, m}, B_{i, m}-$ коэффициенты связи между $i$-ой и $m$-ой модами; $T_{R}$ - параметр рассеяния Рамана; $\omega_{0, i}$ - круговая частота $i$-ой моды; $z$ - координата, направленная вдоль оси волокна; $t$ - время. 
Частный случай совпадения системы связных нелинейных уравнений Шрёдингера с системой уравнений Манакова, записанной с дисперсией до второго порядка, который достигается при выборе коэффициентов $\beta_{3, y}=\beta_{3, y}=0$, и особом выборе для $\gamma_{x}$ и $\gamma_{y}$ при котором $T_{\mathrm{R}}=0$ и $\omega_{0} \rightarrow \infty$. Решение системы уравнений Манакова, как частного случая системы связных нелинейных уравнений Шрёдингера, полученное предложенным методом полностью и качественно и количественно (рисунок . 1,a) совпадают с решением, полученным нами ранее для уравнения Манакова второго рода [4], и другими авторами [5]. Для тестового расчета можно принять данные, приведенные в [2,5], которые опираются на результаты эксперимента: $\alpha=0.2$ дБм $/$ км, $\beta_{1, x}=4.294 \times 10^{-9} \mathrm{c} / \mathrm{M}, \quad \beta_{1, y}=4.290 \times 10^{-9} \mathrm{c} / \mathrm{M}, \quad \beta_{2, x}=$ $3.600 \times 10^{-26} \mathrm{c}^{2} / \mathrm{M}, \beta_{2, y}=3.250 \times 10^{-26} \mathrm{c}^{2} / \mathrm{M}, \beta_{3, y}=\beta_{3, y}=2.750 \times 10^{-41} \mathrm{c}^{3} / \mathrm{M}, \gamma_{x}=\gamma_{y}=$ $3.600 \times 10^{-2}(\mathrm{м} \cdot \mathrm{BT})^{-1}, T_{R}=4.000 \times 10^{-15} \mathrm{c}, \omega_{0}=2.3612 \times 10^{-15} \mathrm{c}^{-1}$ (длина волны $798 \mathrm{нм}$ ). На входе, одиночный чирпированный импульс с формой кривой Гаусса (чирп $\mathrm{C}$ $=-0.4579)$, длительность импульса на половине высоты - 12 фс, с пиковой мощностью $P=1.75 \times 10^{5}$ Вт. На рисунке 1,6 приведены результаты расчетов прохождения импульса при значениях коэффициентов, приведенных выше.
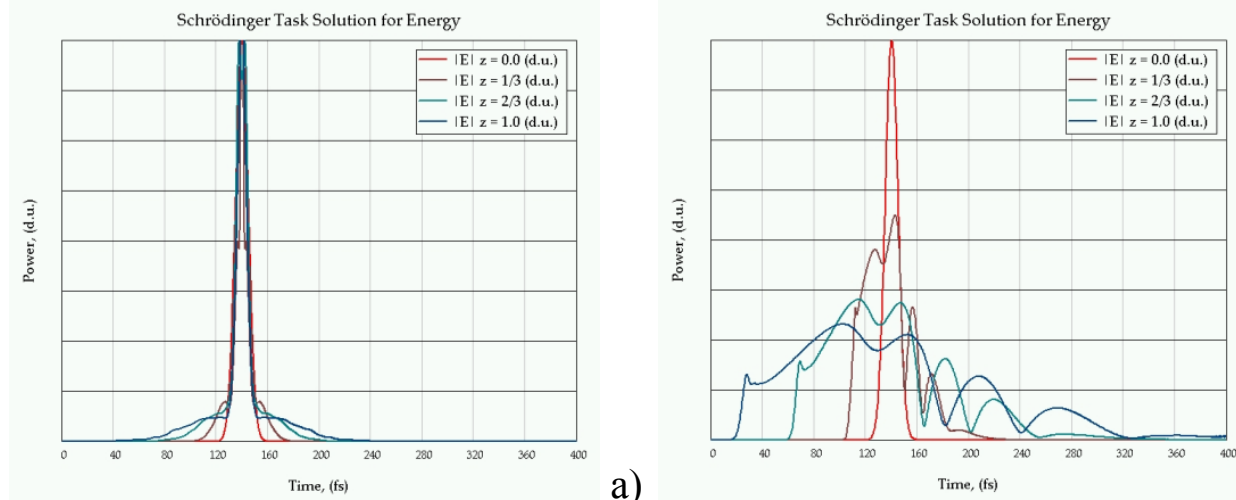

a)

$$
\text { Time, (fs) }
$$

Рис. 1. Изменение формы импульса при распространении его по волокну: а) уравнения Манакова второго рода; б) уравнения Шрёдингера: красная линия при $z=0$ (начальное распределение), коричневая при $z=1 / 3$, зеленая $-z=2 / 3$, синяя при $z=1$ (расстояние 2,5 мм)

Число точек разбиения по времени составило 20 тыс. штук, по расстоянию было выполнено $\sim 720$ тыс. шагов интегрирования, с начальным шагом $\Delta \xi=1 \cdot 10^{-4}$ безразм. ед. и автоматическим регулированием его величины, что позволило просчитать прохождение импульса на $\sim 2.5$ мм. Точность получения решения для итерационного процесса была выбрана $10^{-50}$ безразм. ед. Все вычисления производились с двойной машинной точностью в операционной системе с разрядностью х64. Данные расчетов (синяя линия, 1,6$)$ хорошо согласуются с результатами, приведенными в $[2,4,5]$, что подтверждает работоспособность алгоритма в целом и возможность его применения для решения подобных нелинейных задач.

\section{Литература}

[1] Pouysegur, J., Guichard, F., et al. Proc. Advanced Solid State Lasers, 2015, hal-01359547.

[2] Karasawa, N., Nakamura, S., et al. Nonlinear Opt., 24, 133-138. (2000)

[3] Sakhabutdinov, A.Zh., Anfinogentov, V.I., et al. Computational Technologies.. 25, $42-54$ (2020).

[4] Бурдин, В.А., Бурдин, А.В. Прикладная фотоника, 6, 93-108, (2019)

[5] Sakhabutdinov, A.; Anfinogentov, V.; et al. Fibers, 8, 34, (2020) 